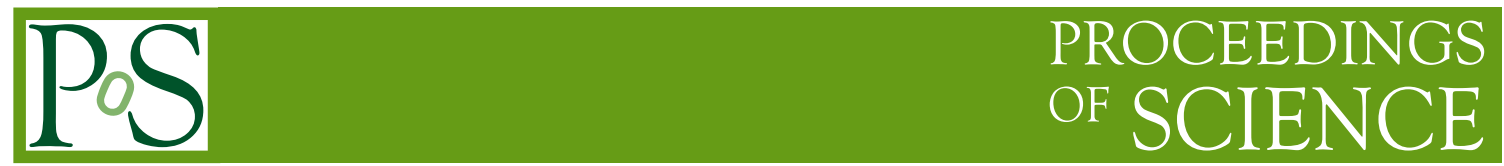

\title{
Covalent hadronic molecules from QCD sum rules
}

\author{
Niu Su, Hua-Xing Chen, ${ }^{*}$ Rui-Rui Dong, Hong-Zhou Xi and Yi-Xin Yan \\ School of Physics, Southeast University, Nanjing 210094, China \\ E-mail: suniu@seu.edu.cn, hxchen@seu.edu.cn, rrdong@seu.edu.cn, \\ hzxi@seu.edu.cn, yxyan@seu.edu.cn
}

We examine Feynman diagrams corresponding to the $\bar{D} \Sigma_{c}$ hadronic molecular state, and propose a possible binding mechanism induced by shared light quarks. We study it using the method of QCD sum rules, and our results indicate this interaction to be attractive as long as the shared light quarks are totally antisymmetric.

*** Particles and Nuclei International Conference - PANIC2021 ***

*** 5 - 10 September, $2021 * * *$

$* * *$ Online $* * *$

${ }^{*}$ Speaker 


\section{Introduction}

Since the discovery of the $X$ (3872) in 2003 by the Belle Collaboration [1], many charmoniumlike $X Y Z$ and hidden-charm $P_{c} / P_{c s}$ states were discovered in the past two decades [2]. They are good candidates of tetraquark and pentaquark states, and their experimental and theoretical studies are significantly improving our understanding of Quantum Chromodynamics (QCD) at the low energy region [3-12]. Some of them can be interpreted as hadronic molecular states consisting of two conventional hadrons [13-15]. For example, the $P_{c}$ states [16] are explained as $\bar{D}^{(*)} \Sigma_{c}^{(*)}$ hadronic molecular states in Refs. [17-22] bound by the one-meson-exchange interaction.

In this paper we propose another possible Feynman diagram between $\bar{D}$ and $\Sigma_{c}$ induced by the light-quark-exchange interaction [23], as depicted in the left panel of Fig. 1. This diagram indicates that $\bar{D}$ and $\Sigma_{c}$ are exchanging two light up/down quarks, which can induce some interaction between them, as depicted in the right panel of Fig. 1. Note that the two interactions, the one-meson-exchange interaction at the hadron level and the light-quark-exchange interaction at the quark-gluon level, can overlap with each other.
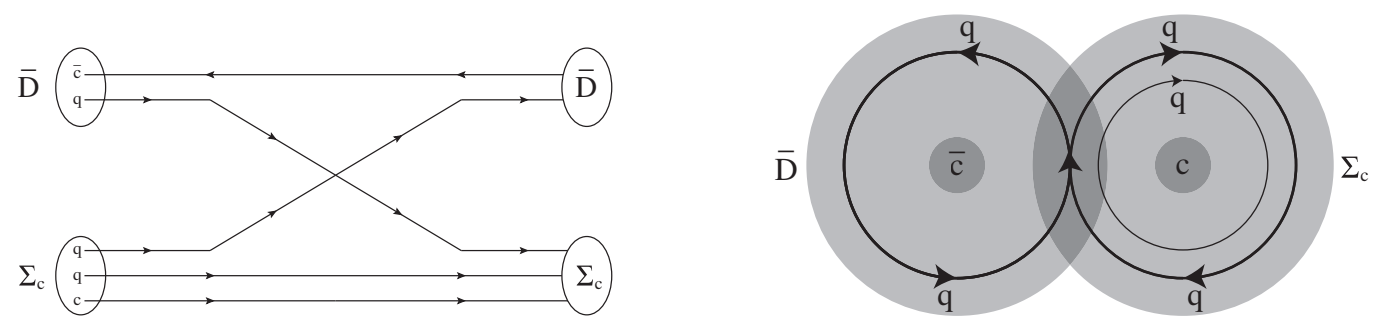

Figure 1: In the left panel we show the Feynman diagram between $\bar{D}$ and $\Sigma_{c}$ corresponding to the lightquark-exchange interaction. In the right panel we show the possible binding mechanism induced by this interaction. Here $q$ denotes a light up/down quark.

In this paper we shall systematically examine Feynman diagrams corresponding to the $\bar{D} \Sigma_{c}$ hadronic molecular state. We shall apply the method of QCD sum rules to investigate the lightquark-exchange interaction, and further propose a model-independent hypothesis: "the light-quarkexchange interaction is attractive when the shared light quarks are totally antisymmetric".

\section{Correlation function of the $\bar{D} \Sigma_{c}$ hadronic molecule}

In this section we investigate correlation functions of the $D^{-} \Sigma_{c}^{++}, \bar{D}^{0} \Sigma_{c}^{+}$, and $I=1 / 2 \bar{D} \Sigma_{c}$ hadronic molecular states. Their corresponding interpolating currents are

$$
\begin{aligned}
J^{D^{-} \Sigma_{c}^{++}}(x) & =\left[\bar{c}_{d}(x) \gamma_{5} d_{d}(x)\right] \times \frac{1}{\sqrt{2}}\left[\epsilon^{a b c} u_{a}^{T}(x) \mathbb{C} \gamma^{\mu} u_{b}(x) \gamma_{\mu} \gamma_{5} c_{c}(x)\right] \\
J^{\bar{D}^{0} \Sigma_{c}^{+}}(x) & =\left[\bar{c}_{d}(x) \gamma_{5} u_{d}(x)\right] \times\left[\epsilon^{a b c} u_{a}^{T}(x) \mathbb{C} \gamma^{\mu} d_{b}(x) \gamma_{\mu} \gamma_{5} c_{c}(x)\right] \\
J_{I=1 / 2}^{\bar{D} \Sigma_{c}}(x) & =\sqrt{\frac{1}{3}} J^{\bar{D}^{0} \Sigma_{c}^{+}}(x)-\sqrt{\frac{2}{3}} J^{D^{-} \Sigma_{c}^{++}}(x) .
\end{aligned}
$$


Their correlation functions in the coordinate space can be separated into:

$$
\begin{aligned}
\Pi^{D^{-} \Sigma_{c}^{++}}(x) & =\Pi_{0}^{\bar{D} \Sigma_{c}}(x)+\Pi_{G}^{\bar{D} \Sigma_{c}}(x), \\
\Pi^{\bar{D} \Sigma_{c}^{+}}(x) & =\Pi_{0}^{\bar{D} \Sigma_{c}}(x)+\Pi_{G}^{\bar{D} \Sigma_{c}}(x)-\Pi_{Q}^{\bar{D} \Sigma_{c}}(x), \\
\Pi_{I=1 / 2}^{\bar{D} \Sigma_{c}}(x) & =\Pi_{0}^{\bar{D} \Sigma_{c}}(x)+\Pi_{G}^{\bar{D} \Sigma_{c}}(x)+\Pi_{Q}^{\bar{D} \Sigma_{c}}(x),
\end{aligned}
$$

where $\Pi_{0}^{\bar{D} \Sigma_{c}}(x)=\Pi^{\bar{D}}(x) \times \Pi^{\Sigma_{c}}(x)$ is the leading term contributed by non-correlated $\bar{D}$ and $\Sigma_{c}$; $\Pi_{G}^{\bar{D} \Sigma_{c}}(x)$ describes the double-gluon-exchange interaction between $\bar{D}$ and $\Sigma_{c}$, which is not taken into account in the present study; $\Pi_{Q}^{\bar{D} \Sigma_{c}}(x)$ describes the light-quark-exchange interaction between $\bar{D}$ and $\Sigma_{c}$.

\section{QCD sum rule study of the $\bar{D} \Sigma_{c}$ hadronic molecule}

In this section we apply the method of QCD sum rules to investigate the light-quark-exchange interaction $\Pi_{Q}(x)$, and study its contributions to the $D^{-} \Sigma_{c}^{++}, \bar{D}^{0} \Sigma_{c}^{+}$, and $I=1 / 2 \bar{D} \Sigma_{c}$ hadronic molecular states.

Given $X \equiv\left|\bar{D} \Sigma_{c}\right\rangle$ to be a molecular state, its mass $M_{X}$ can be expanded as

$$
M_{X}=M_{\bar{D}}+M_{\Sigma_{c}}+\Delta M \equiv M_{0}+\Delta M
$$

so that we can expand its correlation function at the hadron level as

$$
\Pi\left(q^{2}\right)=\frac{f_{X}^{2}}{M_{X}^{2}-q^{2}}+\cdots \approx \frac{f_{X}^{2}}{M_{0}^{2}-q^{2}}-\frac{2 M_{0} f_{X}^{2}}{\left(M_{0}^{2}-q^{2}\right)^{2}} \Delta M+\cdots .
$$

The former is contributed by non-correlated $\bar{D}$ and $\Sigma_{c}$, and the latter is contributed by their interactions. After comparing Eq. (4) to Eq. (2), we perform the Borel transformation at both hadron and quark-gluon levels to obtain:

$$
\begin{aligned}
f_{X}^{2} e^{-M_{0}^{2} / M_{B}^{2}} & =\Pi_{0}\left(M_{B}^{2}, s_{0}\right)=\int_{s_{<}}^{s_{0}} e^{-s / M_{B}^{2}} \rho_{0}(s) d s, \\
-\frac{2 M_{0} f_{X}^{2}}{M_{B}^{2}} \Delta M e^{-M_{0}^{2} / M_{B}^{2}} & =\Pi_{Q}\left(M_{B}^{2}, s_{0}\right)=\int_{s_{<}}^{s_{0}} e^{-s / M_{B}^{2}} \rho_{Q}(s) d s,
\end{aligned}
$$

which can be used to further derive

$$
-\frac{2 M_{0}}{M_{B}^{2}} \Delta M=\frac{\Pi_{Q}\left(M_{B}^{2}, s_{0}\right)}{\Pi_{0}\left(M_{B}^{2}, s_{0}\right)}=\frac{\int_{s_{<}}^{s_{0}} e^{-s / M_{B}^{2}} \rho_{Q}(s) d s}{\int_{s_{<}}^{s_{0}} e^{-s / M_{B}^{2}} \rho_{0}(s) d s} .
$$

\section{Discussions on the parameter $\Delta M$}

The parameter $\Delta M$ is actually not the binding energy, because we are using local currents in QCD sum rule analyses. We can relate it to some potential $V(r)$ between $\bar{D}$ and $\Sigma_{c}$, satisfying $V(r=0)=\Delta M$ and $V(r \rightarrow \infty) \rightarrow 0$. We systematically investigate the light-quark-exchange 
interaction $\Pi_{Q}(x)$, and study its contributions to the $D^{-} \Sigma_{c}^{++}, \bar{D}^{0} \Sigma_{c}^{+}$, and $I=1 / 2 \bar{D} \Sigma_{c}$ hadronic molecular states. Their mass corrections are evaluated to be:

$$
\begin{aligned}
\Delta M^{D^{-} \Sigma_{c}^{++}} & =0 \\
\Delta M^{\bar{D}^{0} \Sigma_{c}^{+}} & =95 \mathrm{MeV} \\
\Delta M_{I=1 / 2}^{\bar{D} \Sigma_{c}} & =-95 \mathrm{MeV} .
\end{aligned}
$$

Accordingly, our results suggest that there can be the $\bar{D} \Sigma_{c}$ covalent molecule of $I=1 / 2$. Its binding mechanism induced by shared light quarks is similar to the covalent bond in the chemical molecule induced by shared electrons, so we call such hadronic molecule the "covalent hadronic molecule".

Recalling that the two shared electrons must spin in opposite directions when forming a chemical covalent bond, our QCD sum rule results indicate a similar hypothesis: "the light-quarkexchange interaction is attractive when the shared light quarks are totally antisymmetric".

\section{Acknowledgments}

This project is supported by the National Natural Science Foundation of China under Grant No. 11722540 and No. 12075019, the Jiangsu Provincial Double-Innovation Program under Grant No. JSSCRC2021488, and the Fundamental Research Funds for the Central Universities.

\section{References}

[1] S. K. Choi et al. [Belle Collaboration], Observation of a Narrow Charmoniumlike State in Exclusive $B^{ \pm} \rightarrow K^{ \pm} \pi^{+} \pi^{-} J / \psi$ Decays, Phys. Rev. Lett. 91, 262001 (2003) [arXiv:hepex/0309032 [hep-ex]].

[2] P. A. Zyla et al. [Particle Data Group], Review of Particle Physics, PTEP 2020, 083 C01 (2020).

[3] H. X. Chen, W. Chen, X. Liu and S. L. Zhu, The hidden-charm pentaquark and tetraquark states, Phys. Rept. 639, 1 (2016) [arXiv:1601.02092 [hep-ph]].

[4] Y. R. Liu, H. X. Chen, W. Chen, X. Liu and S. L. Zhu, Pentaquark and Tetraquark States, Prog. Part. Nucl. Phys. 107, 237 (2019) [arXiv:1903.11976 [hep-ph]].

[5] A. Hosaka, T. Iijima, K. Miyabayashi, Y. Sakai and S. Yasui, Exotic hadrons with heavy flavors: X, Y, Z, and related states, PTEP 2016, $062 \mathrm{C01}$ (2016) [arXiv:1603.09229 [hep-ph]].

[6] R. F. Lebed, R. E. Mitchell and E. S. Swanson, Heavy-quark QCD exotica, Prog. Part. Nucl. Phys. 93, 143 (2017) [arXiv:1610.04528 [hep-ph]].

[7] A. Esposito, A. Pilloni and A. D. Polosa, Multiquark resonances, Phys. Rept. 668, 1 (2017) [arXiv:1611.07920 [hep-ph]].

[8] A. Ali, J. S. Lange and S. Stone, Exotics: Heavy pentaquarks and tetraquarks, Prog. Part. Nucl. Phys. 97, 123 (2017) [arXiv:1706.00610 [hep-ph]]. 
[9] F. K. Guo, C. Hanhart, U. G. Meißner, Q. Wang, Q. Zhao, and B. S. Zou, Hadronic molecules, Rev. Mod. Phys. 90, 015004 (2018) [arXiv:1705.00141 [hep-ph]].

[10] S. L. Olsen, T. Skwarnicki, and D. Zieminska, Nonstandard heavy mesons and baryons: Experimental evidence, Rev. Mod. Phys. 90, 015003 (2018) [arXiv:1708.04012 [hep-ph]].

[11] M. Karliner, J. L. Rosner and T. Skwarnicki, Multiquark States, Ann. Rev. Nucl. Part. Sci. 68, 17 (2018) [arXiv:1711.10626 [hep-ph]].

[12] N. Brambilla, S. Eidelman, C. Hanhart, A. Nefediev, C. P. Shen, C. E. Thomas, A. Vairo and C. Z. Yuan, The XYZ states: experimental and theoretical status and perspectives, Phys. Rept. 873, 1 (2020) [arXiv:1907.07583 [hep-ex]].

[13] S. Weinberg, Evidence That the Deuteron Is Not an Elementary Particle, Phys. Rev. 137, B672 (1965).

[14] M. B. Voloshin and L. B. Okun, Hadron Molecules and Charmonium Atom, JETP Lett. 23, 333 (1976) [Pisma Zh. Eksp. Teor. Fiz. 23, 369 (1976)].

[15] A. De Rujula, H. Georgi and S. L. Glashow, Molecular Charmonium: A New Spectroscopy? Phys. Rev. Lett. 38, 317 (1977).

[16] R. Aaij et al. [LHCb Collaboration], Observation of $J / \psi p$ Resonances Consistent with Pentaquark States in $\Lambda_{b}^{0} \rightarrow J / \psi K^{-} p$ Decays, Phys. Rev. Lett. 115, 072001 (2015) [arXiv: 1507.03414 [hep-ex]].

[17] J. J. Wu, R. Molina, E. Oset and B. S. Zou, Prediction of Narrow $N^{*}$ and $\Lambda^{*}$ Resonances with Hidden Charm above 4 GeV, Phys. Rev. Lett. 105, 232001 (2010) [arXiv:1007.0573 [nucl-th]].

[18] Z. C. Yang, Z. F. Sun, J. He, X. Liu and S. L. Zhu, Possible hidden-charm molecular baryons composed of an anti-charmed meson and a charmed baryon, Chin. Phys. C 36, 6 (2012) [arXiv:1105.2901 [hep-ph]].

[19] M. Karliner and J. L. Rosner, New Exotic Meson and Baryon Resonances from Doubly-Heavy Hadronic Molecules, Phys. Rev. Lett. 115, 122001 (2015) [arXiv:1506.06386 [hep-ph]].

[20] R. Chen, X. Liu, X. Q. Li and S. L. Zhu, Identifying exotic hidden-charm pentaquarks, Phys. Rev. Lett. 115, 132002 (2015) [arXiv:1507.03704 [hep-ph]].

[21] H. X. Chen, W. Chen, X. Liu, T. G. Steele and S. L. Zhu, Towards exotic hidden-charm pentaquarks in QCD, Phys. Rev. Lett. 115, 172001 (2015) [arXiv:1507.03717 [hep-ph]].

[22] M. Z. Liu, Y. W. Pan, F. Z. Peng, M. S. Sánchez, L. S. Geng, A. Hosaka and M. P. Valderrama, Emergence of a Complete Heavy-Quark Spin Symmetry Multiplet: Seven Molecular Pentaquarks in Light of the Latest LHCb Analysis, Phys. Rev. Lett. 122, 242001 (2019) [arXiv:1903.11560 [hep-ph]].

[23] H. X. Chen, Covalent hadronic molecules induced by shared light quarks, arXiv:2105.09193 [hep-ph]. 